\title{
Multiplicity result for a stationary fractional reaction-diffusion equations
}

\author{
César E. Torres Ledesma \\ Departamento de Mathemáticas, Universidad Nacional de Trujillo Av. Juan Pablo II s/n Trujillo, Peru \\ E-mail: ctl_576@yahoo.es
}

\begin{abstract}
In this paper, we consider the stationary fractional reaction-diffusion equations with RiemannLiouville boundary conditions

$$
\begin{aligned}
& { }_{x} D_{T}^{\alpha}\left({ }_{0} D_{x}^{\alpha} u(x)\right)+{ }_{0} D_{x}^{\beta}\left({ }_{x} D_{T}^{\beta} u(x)\right)=f(x, u(x)), \quad x \in(0, T), \\
& \lim _{x \rightarrow 0}{ }_{0} I_{x}^{1-\alpha} u(x)=\lim _{x \rightarrow T}{ }_{x} I_{T}^{1-\beta} u(x)=0 .
\end{aligned}
$$

where $0<\alpha, \beta<1$ and $f \in C([0, T] \times \mathbb{R}, \mathbb{R})$. Under suitable conditions on the nonlinearity $f$, we study the multiplicity of weak solutions of $(0.1)$ by using the genus in the critical point theory.
\end{abstract}

2010 Mathematics Subject Classification. 26A33. 34A08, 30E25

Keywords. Riemann-Liouville fractional derivatives, fractional derivative space, boundary value problem, genus, variational methods.

\section{Introduction}

Fractional differential equations have been an area of great interest recently. This is because of both the intensive development of the theory of fractional calculus itself and the applications of such constructions in various scientific fields such as physics, chemistry, biology, geology, as well as, control theory, signal theory, nanoscience and so on $[3,14,18,21,22,34]$ and references therein. In fact, the adequacy of fractional derivatives to describe the memory effects and hereditary properties in a great variety of processes makes fractional differential models interesting and with a great potential in applications, which is supported by the good adjustment between simulations and experimental data. As indicated in [20], the dynamics of natural systems are in many occasions complex, so that classical models might not be adequate. In this reference, the authors also show that the order of the fractional derivative is important to control the speed in which the trajectories of fractional systems move with respect to the critical point. This behavior is found by the authors of [20] as an interesting issue in relation with anomalous behavior appearing among competing species or in the study of diseases and justifies the applicability of fractional models in biology.

The existence and multiplicity of solutions for BVP for nonlinear fractional differential equations is extensively studied using various tools of nonlinear analysis as fixed point theorems, degree theory and the method of upper and lower solutions $[4,5]$. Very recently, it should be noted that critical point theory and variational methods have also turned out to be very effective tools in determining the existence of solutions of BVP for fractional differential equations. The idea behind them is trying to find solutions of a given boundary value problem by looking for critical points of a suitable energy functional defined on an appropriate function space. In the last 30 years, the critical point 
theory has become a wonderful tool in studying the existence of solutions to differential equations with variational structures, we refer the reader to the books due to Mawhin and Willem [15], Rabinowitz [19], Schechter [23] and papers [10, 12, 13, 17, 26, 27, 28, 31] for bounded intervals and $[16,25,29,30,33,34]$ in the real line.

Very recently Fazli and Bahrami [11], have study the existence of weak solutions to the steady fractional reaction-diffusion equation

$$
{ }_{x} D_{1}^{\alpha}\left({ }_{0} D_{x}^{\alpha} y(x)\right)+{ }_{0} D_{x}^{\beta}\left({ }_{x} D_{1}^{\beta} y(x)\right)=f(x, y(x))
$$

with Riemann-Liouville boundary conditions

$$
\lim _{x \rightarrow 0}{ }_{0} I_{x}^{1-\alpha} y(x)=\lim _{x \rightarrow 1} I_{1}^{1-\beta} y(x)=0,
$$

where $0<\alpha, \beta<1, f:[0,1] \times \mathbb{R} \rightarrow \mathbb{R}$ is Carathéodory function and ${ }_{0} D_{x}^{\alpha},{ }_{x} D_{1}^{\alpha}$ denote left and right Riemann-Liouville fractional derivatives of order $\alpha$ respectively. Under some suitable conditions on $f$, the authors show the existence results by using generalized Weierstrass theorem and relatively simple techniques from nonlinear functional analysis.

Equation (1.1) comes from the fractional reaction-diffusion equation

$$
\frac{\partial}{\partial t} y(t, x)={ }_{x} D_{1}^{\alpha}\left({ }_{0} D_{x}^{\alpha} y(x)\right)+{ }_{0} D_{x}^{\beta}\left({ }_{x} D_{1}^{\beta} y(x)\right)+f(x, y(x)),
$$

that is a generalization of the classical reaction-diffusion equation in which the second-order derivative is replaced with a fractional derivative of order less than two. The resulting solutions spread faster than the classical solutions and may exhibit asymmetry, depending on the fractional derivative used, see $[6,8,24]$ for more details.

In this paper, we consider the multiplicity result for $(1.1)$ over $(0, T)$, namely, we consider the following fractional boundary value problem:

$$
\begin{aligned}
& { }_{x} D_{T}^{\alpha}\left({ }_{0} D_{x}^{\alpha} u(x)\right)+{ }_{0} D_{x}^{\beta}\left({ }_{x} D_{T}^{\beta} u(x)\right)=f(x, u(x)), \quad x \in(0, T), \\
& \lim _{x \rightarrow 0}{ }_{0} I_{x}^{1-\alpha} u(x)=\lim _{x \rightarrow T}{ }_{x} I_{T}^{1-\beta} u(x)=0 .
\end{aligned}
$$

where $0<\alpha, \beta<1$ and $f \in C([0, T] \times \mathbb{R}, \mathbb{R})$ satisfy the following assumptions

$\left(f_{1}\right) F(t, 0)=0$ for all $t \in[0, T], F(t, u) \geq a(t)|u|^{q}$ and $|f(t, u)| \leq q b(t)|u|^{q-1}$ for all $(t, u) \in$ $[0, T] \times \mathbb{R}$, where $1<q<2$ is a constant, $a, b:[0, T] \rightarrow \mathbb{R}^{+}$are a continuous functions and $F(t, u)=\int_{0}^{u} f(t, s) d s$.

$\left(f_{2}\right)$ There is a constant $1<\mu \leq q<2$ such that

$$
f(t, u) u \leq \mu F(t, u) \text { for all } t \in[0, T] \text { and } u \in \mathbb{R} .
$$

$\left(f_{3}\right) F(t, u)=F(t,-u)$ for all $t \in[0, T]$ and $u \in \mathbb{R}$.

Before stating our result let us introduce the main ingredients involved in our approach. We let $\mathbb{H}_{0}^{\alpha, \beta}(0, T)$ be the fractional Hilbert space defined by

$$
\mathbb{H}_{0}^{\alpha, \beta}(0, T):=\left\{u \in L^{2}(0, T):{ }_{0} D_{x}^{\alpha} u,{ }_{x} D_{T}^{\beta} u \in L^{2}(0, T), \quad \lim _{x \rightarrow 0^{+}}{ }_{0} I_{x}^{1-\alpha} u(x)=\lim _{x \rightarrow T^{-}}{ }_{x} I_{T}^{1-\beta} u(x)=0\right\}
$$


equipped with the norm

$$
\|u\|_{\alpha, \beta}=\left(\int_{0}^{T}\left|{ }_{0} D_{x}^{\alpha} u(x)\right|^{2} d x+\left.\int_{0}^{T}{ }_{x} D_{T}^{\beta} u(x)\right|^{2} d x\right)^{1 / 2}
$$

For $u \in \mathbb{H}_{0}^{\alpha, \beta}(0, T)$ and $f$ satisfying $\left(f_{1}\right)$, as we see in Sect. $\S 3$, we may define the functional $I: \mathbb{H}_{0}^{\alpha, \beta}(0, T) \rightarrow \mathbb{R}$ by

$$
I(u)=\frac{1}{2}\|u\|_{\alpha, \beta}^{2}-\int_{0}^{T} F(t, u(t)) d t,
$$

which is of class $C^{1}$. Moreover, we say that $u \in \mathbb{H}_{0}^{\alpha, \beta}(0, T)$ is a weak solution of (1.3) if $u$ is a critical point of $I$.

Now we are in position to state our main result.

Theorem 1.1. Suppose that $\left(f_{1}\right)-\left(f_{3}\right)$ are satisfied. Then, (1.1) has infinitely many nontrivial solutions.

The rest of the paper is organized as follows: In Section $\S 2$ we present preliminaries on fractional calculus and we introduce the functional setting of the problem. In Section $\S 3$ we prove Theorem 1.1 .

\section{Preliminary results}

In this section we introduce some basic definitions of fractional calculus which are used further in this paper. For the proof see $[14,18,22]$.

Let $u$ be a function defined on $[a, b]$. The left (right ) Riemann-Liouville fractional integral of order $\alpha>0$ for function $u$ is defined by

$$
\begin{aligned}
& { }_{a} I_{x}^{\alpha} u(x)=\frac{1}{\Gamma(\alpha)} \int_{a}^{x}(x-t)^{\alpha-1} u(t) d t, x \in[a, b], \\
& { }_{x} I_{b}^{\alpha} u(x)=\frac{1}{\Gamma(\alpha)} \int_{x}^{b}(t-x)^{\alpha-1} u(t) d t, x \in[a, b],
\end{aligned}
$$

provided in both cases that the right-hand side is pointwise defined on $[a, b]$. Here and in what follows $\Gamma$ denotes the Gamma function.

The left and right Riemann - Liouville fractional derivatives of order $\alpha \in(0,1]$ of a function $u$ denoted by ${ }_{a} D_{t}^{\alpha} u(t)$ and ${ }_{t} D_{b}^{\alpha} u(t)$, respectively, are defined by

$$
\begin{gathered}
{ }_{a} D_{x}^{\alpha} u(x)=\frac{d}{d x} a I_{x}^{1-\alpha} u(x), \\
{ }_{x} D_{b}^{\alpha} u(x)=-\frac{d}{d x}{ }_{x} I_{b}^{1-\alpha} u(x),
\end{gathered}
$$

where $x \in[a, b]$.

Now we consider some properties of the Riemann-Liouville fractional integral and derivative operators [14].

Theorem 2.1. 1. We have

$$
{ }_{a} I_{x}^{\alpha}\left({ }_{a} I_{x}^{\beta} u(x)\right)={ }_{a} I_{x}^{\alpha+\beta} u(x), \quad{ }_{x} I_{b}^{\alpha}\left({ }_{x} I_{b}^{\beta} u(x)\right)={ }_{x} I_{b}^{\alpha+\beta} u(x), \quad \forall \alpha, \beta>0,
$$


2. Left inverse. Let $u \in L^{1}[a, b]$ and $\alpha>0$,

$$
{ }_{a} D_{x}^{\alpha}\left({ }_{a} I_{x}^{\alpha} u(x)\right)=u(x) \text {, a.e. } t \in[a, b],{ }_{x} D_{b}^{\alpha}\left({ }_{x} I_{b}^{\alpha} u(x)\right)=u(x) \text {, a.e. } x \in[a, b] .
$$

3. Let $\alpha \in(0,1]$ and $p \geq 1$. If ${ }_{a} D_{x}^{\alpha} u(x) \in L^{p}(a, b)\left(\operatorname{resp.}{ }_{x} D_{b}^{\alpha} u(x) \in L^{p}(a, b)\right)$, then

$$
\begin{aligned}
& { }_{a} I_{x a}^{\alpha} D_{x}^{\alpha} u(x)=u(x)-\frac{{ }_{a} I_{x}^{1-\alpha} u(a)}{\Gamma(\alpha)}(x-a)^{\alpha-1} \text { and } \\
& { }_{x} I_{T}^{\alpha}{ }^{\alpha} D_{T}^{\alpha} u(x)=u(x)-\frac{{ }_{x} I_{b}^{1-\alpha} u(b)}{\Gamma(\alpha)}(b-x)^{\alpha-1}
\end{aligned}
$$

4. Let $p \geq 1$. The fractional integration operators ${ }_{a} I_{x}^{\alpha}$ and ${ }_{x} I_{b}^{\alpha}$ with $\alpha>0$ are bounded in $\left.L^{p}(a, b)\right]$ and

$$
\left\|_{a} I_{x}^{\alpha} u\right\|_{L^{p}} \leq \frac{(b-a)^{\alpha}}{\Gamma(\alpha+1)}\|u\|_{L^{p}}, \quad\left\|I_{x}^{\alpha}\right\|_{L^{p}} \leq \frac{(b-a)^{\alpha}}{\Gamma(\alpha+1)}\|u\|_{L^{p}}
$$

\section{Integration by parts}

$$
\int_{a}^{b}\left[{ }_{a} I_{x}^{\alpha} u(x)\right] v(x) d x=\int_{a}^{b} u(x)_{x} I_{b}^{\alpha} v(x) d x, \alpha>0,
$$

provided that $u \in L^{p}[a, b], v \in L^{q}[a, b]$ and

$$
p \geq 1, q \geq 1 \text { and } \frac{1}{p}+\frac{1}{q}<1+\alpha \text { or } p \neq 1, q \neq 1 \text { and } \frac{1}{p}+\frac{1}{q}=1+\alpha .
$$

6. Let $0<\frac{1}{p}<\alpha \leq 1$ and $u(x) \in L^{p}[0, T]$, then ${ }_{0} I_{t}^{\alpha} u$ is Hölder continuous on $[0, T]$ with exponent $\alpha-\frac{1}{p}$ and $\lim _{x \rightarrow 0^{+}}{ }_{0} I_{x}^{\alpha} u(x)=0$. Consequently, ${ }_{0} I_{x}^{\alpha} u$ can be continuously extended by 0 in $x=0$.

\subsection{Fractional Hilbert space}

In this section we introduce the fractional Hilbert space in which boundary conditions are nonlocal, we follow [11]. For $\alpha, \beta \in(0,1)$, we define the following fractional space

$$
\mathbb{H}_{0}^{\alpha, \beta}:=\left\{u \in L^{2}(0, T): \quad{ }_{0} D_{x}^{\alpha} u,{ }_{x} D_{T}^{\beta} u \in L^{2}(0, T), \quad \lim _{x \rightarrow 0^{+}}{ }_{0} I_{x}^{1-\alpha} u(x)=\lim _{x \rightarrow T^{-}}{ }_{x} I_{T}^{1-\beta} u(x)=0\right\}
$$

with the inner product

$$
\langle u, v\rangle_{\alpha, \beta}=\int_{0}^{T}{ }_{0} D_{x}^{\alpha} u(x) \cdot{ }_{0} D_{x}^{\alpha} v(x) d x+\int_{0}^{T}{ }_{x} D_{T}^{\beta} u(x) \cdot{ }_{x} D_{T}^{\beta} v(x) d x,
$$

and the corresponding norm

$$
\|u\|_{\alpha, \beta}:=\left(\left.\left.\int_{0}^{T}\right|_{0} D_{x}^{\alpha} u(x)\right|^{2} d x+\left.\int_{0}^{T}{ }_{x} D_{T}^{\beta} u(x)\right|^{2} d x\right)^{1 / 2} .
$$

Clearly, if $\alpha=\beta=1$, the space $\mathbb{H}_{0}^{\alpha, \beta}(0, T)$ coincides with the Sobolev space $H_{0}^{1}(0, T)$. 
Remark 2.2. By Theorem $2.1-3$ and 4 , for every $u \in \mathbb{H}_{0}^{\alpha, \beta}(0, T)$, we have

$$
{ }_{0} I_{x 0}^{\alpha} D_{x}^{\alpha} u(x)=u(x) \text { and }{ }_{x} I_{T x}^{\beta} D_{T}^{\beta} u(x)=u(x),
$$

next

$$
\|u\|_{L^{2}}=\left\|_{0} I_{x 0}^{\alpha} D_{x}^{\alpha} u\right\|_{L^{2}} \leq \frac{T^{\alpha}}{\Gamma(\alpha+1)}\left\|_{0} D_{x}^{\alpha} u\right\|_{L^{2}}
$$

and

$$
\|u\|_{L^{2}}=\left\|_{x} I_{T^{x}}^{\beta} D_{T}^{\beta} u\right\|_{L^{2}} \leq \frac{T^{\beta}}{\Gamma(\beta+1)}\left\|_{x} D_{T}^{\beta} u\right\|_{L^{2}}
$$

Therefore

$$
\|u\|_{L^{2}} \leq \mathfrak{C}\left(\left\|D_{0} D_{x}^{\alpha} u\right\|_{L^{2}}^{2}+\left\|_{x} D_{T}^{\beta} u\right\|_{L^{2}}^{2}\right)^{1 / 2}=\mathfrak{C}\|u\|_{\alpha, \beta}
$$

where $\mathfrak{C}=\frac{T^{\alpha}}{\Gamma(\alpha+1)}+\frac{T^{\beta}}{\Gamma(\beta+1)}$. So, the continuous embedding $\mathbb{H}_{0}^{\alpha, \beta}(0, T) \hookrightarrow L^{2}(0, T)$ holds.

In the same way of [11], we can show that for $\alpha, \beta \in(0,1), \mathbb{H}_{0}^{\alpha, \beta}(0, T)$ is a Hilbert space. In fact, let $\left\{u_{n}\right\}$ be a Cauchy sequence in $\mathbb{H}_{0}^{\alpha, \beta}(0, T)$, then $\left\{u_{n}\right\},\left\{{ }_{0} D_{x}^{\alpha} u_{n}\right\}$ and $\left\{{ }_{x} D_{T}^{\beta} u_{n}\right\}$ are Cauchy sequences in $L^{2}(0, T)$. Then

$$
u_{n} \rightarrow u,{ }_{0} D_{x}^{\alpha} u_{n} \rightarrow u^{(\alpha)}, \quad{ }_{x} D_{T}^{\beta} u_{n} \rightarrow u^{(\beta)} \text { in } L^{2}(0, T) .
$$

Following the same argument as in Remark 2.2, we obtain

$$
u_{n} \rightarrow{ }_{0} I_{x}^{\alpha} u^{(\alpha)}(x), \quad u_{n} \rightarrow{ }_{x} I_{T}^{\beta} u^{(\beta)}(x) \text { in } L^{2}(0, T) .
$$

Therefore, we have

$$
u(x)={ }_{0} I_{x}^{\alpha} u^{(\alpha)}(x), \quad u(x)={ }_{x} I_{T}^{\beta} u^{(\beta)}(x) \quad \text { a.e. in }(0, T) .
$$

Furthermore, by Theorem 2.1 - 2, we immediately obtain that $u^{(\alpha)}(x)={ }_{0} D_{x}^{\alpha} u(x), u^{(\beta)}(x)=$ ${ }_{x} D_{T}^{\beta} u(x)$. On the other hand, from (2.6), we have

$$
u(x)={ }_{0} I_{x 0}^{\alpha} D_{x}^{\alpha} u(x), \quad u(x)={ }_{x} I_{T x}^{\beta} D_{T}^{\beta} u(x) \text { a.e. in }[0, T] .
$$

Therefore, using Theorem 2.1 - 3, we deduce

$$
\lim _{x \rightarrow 0^{+}}{ }_{0} I_{x}^{1-\alpha} u(x)=\lim _{x \rightarrow T^{-}} I_{T}^{1-\beta} u(x)=0 .
$$

Furthermore, we have the following compact embedding theorem.

Theorem 2.3. Let $\alpha, \beta \in(0,1)$. Then $\mathbb{H}_{0}^{\alpha, \beta}(0, T) \hookrightarrow L^{2}(0, T)$ is compact.

Remark 2.4. 1. We recall that, every Hilbert space $H$ is uniformly convex, in fact, given $x, y \in$ $B_{H}$ and $0<\varepsilon \leq 2$ such that $\|x-y\| \geq \varepsilon$, then by the parallelogram law, we obtain

$$
\left\|\frac{x+y}{2}\right\|^{2}=\frac{\|x\|^{2}}{2}+\frac{\|y\|^{2}}{2}-\frac{\|x-y\|^{2}}{4} \leq 1-\frac{\varepsilon^{2}}{4} .
$$

So, taking $\delta=1-\left(1-\frac{\varepsilon^{2}}{4}\right)^{1 / 2}>0$ we conclude. Therefore, since $\mathbb{H}_{0}^{\alpha, \beta}(0, T)$ is a Hilbert space, then it is uniformly convex. So by Theorem 1.21 in $[1], \mathbb{H}_{0}^{\alpha, \beta}(0, T)$ is a reflexive space. 
2. We claim that $\mathbb{H}_{0}^{\alpha, \beta}(0, T)$ is separable. In fact, since $L^{2}(0, T)$ is separable, then the product space

$$
L_{2}^{2}(0, T)=L^{2}(0, T) \times L^{2}(0, T)
$$

is also separable space with respect to the norm

$$
\|v\|_{L_{2}^{2}}=\left(\sum_{i=1}^{2}\left\|v_{i}\right\|_{L^{2}}^{2}\right)^{1 / 2}
$$

where $v=\left(v_{1}, v_{2}\right) \in L_{2}^{2}(0, T)$. Now, consider the space $\Gamma=\left\{\left({ }_{0} D_{x}^{\alpha} u,{ }_{x} D_{T}^{\beta} u\right): \quad \forall u \in\right.$ $\left.\mathbb{H}_{0}^{\alpha, \beta}(0, T)\right\}$, which is a closed subset of $L_{2}^{2}(0, T)$ as $\mathbb{H}_{0}^{\alpha, \beta}(0, T)$ is closed. So $\Gamma$ is also separable space with respect to the norm $(2.7)$ for $v=\left(v_{1}, v_{2}\right) \in \Gamma$. Consider the operator $B: \mathbb{H}_{0}^{\alpha, \beta}(0, T) \rightarrow \Gamma$ defined by

$$
B: u \rightarrow\left({ }_{0} D_{x}^{\alpha} u,{ }_{x} D_{T}^{\beta} u\right), \quad \forall u \in \mathbb{H}_{0}^{\alpha, \beta}(0, T)
$$

Then

$$
\|u\|_{\alpha, \beta}=\|B u\|_{L_{2}^{2}}
$$

which means that the operator $B$ is an isometric mapping and the space $\mathbb{H}_{0}^{\alpha, \beta}(0, T)$ is isometric isomorphic to the space $\Gamma$. Therefore $\mathbb{H}_{0}^{\alpha, \beta}(0, T)$ is separable Hilbert space.

Now we introduce more notations and some necessary definitions. Let $\mathfrak{B}$ be a real Banach space, $I \in C^{1}(\mathfrak{B}, \mathbb{R})$ means that $I$ is a continuously Fréchet differentiable functional defined on $\mathfrak{B}$.

Definition 2.5. $I \in C^{1}(\mathcal{B}, \mathbb{R})$ is said to satisfy the (PS) condition if any sequence $\left\{u_{j}\right\}_{j \in \mathbb{N}} \subset \mathcal{B}$, for which $\left\{I\left(u_{j}\right)\right\}_{j \in \mathbb{N}}$ is bounded and $I^{\prime}\left(u_{j}\right) \rightarrow 0$ as $j \rightarrow+\infty$, possesses a convergent subsequence in $\mathcal{B}$.

In order to find infinitely many solutions of (1.1), we will use the Krasnoselskii genus. Let us denote by $\Sigma$ the class of all closed subsets $A \in \mathfrak{B} \backslash\{0\}$ that are symmetric with respect to the origin, that is, $u \in A$ implies $-u \in A$.

Definition 2.6. Let $A \in \Sigma$. The Krasnoselskii genus $\gamma(A)$ of $A$ is defined as being the least positive integer $k$ such that there is an odd mapping $\varphi \in C\left(A, \mathbb{R}^{k}\right)$ such that $\varphi(x) \neq 0$ for all $x \in A$. If such a $k$ does not exist we set $\gamma(A)=\infty$. Furthermore, by definition, $\gamma(\varnothing)=0$.

In the sequel, we will state only the properties of the genus that will be used through this work. More information on this subject may be found in [2].

Theorem 2.7. Let $\mathfrak{B}=\mathbb{R}^{n}$ and $\partial \Omega$ be the boundary of an open, symmetric and bounded subset $\Omega \in \mathbb{R}^{n}$ with $0 \in \Omega$. Then $\gamma(\partial \Omega)=n$.

Corollary 2.8. $\gamma\left(S^{n-1}\right)=n$.

As a consequence of this, if $\mathfrak{B}$ is of infinite dimension and separable and $S$ is the unit sphere in $\mathfrak{B}$, then $\gamma(S)=\infty$. We now state a result due to Clarke [9] 
Theorem 2.9. Let $I \in C^{1}(\mathfrak{B}, \mathbb{R})$ be a functional satisfying the (PS)-condition. Furthermore, let us suppose that:

(i) $I$ is bounded from below and even,

(ii) there is a compact set $K \in \Sigma$ such that $\gamma(K)=k$ and $\sup _{u \in K} I(u)<0$.

Then $I$ possesses at least $k$ pairs of distinct critical points and their corresponding critical values are less than $I(0)$.

\section{Multiplicity result}

In this section we are going to prove Theorem 1.1. We start our analysis with the following Lemma.

Lemma 3.1. Assume that $\left(f_{1}\right)$ hold. Then the functional $I$ is well defined and of class $C^{1}\left(\mathbb{H}_{0}^{\alpha, \beta}(0, T), \mathbb{R}\right)$ and

$$
\left\langle I^{\prime}(u), v\right\rangle=\int_{0}^{T}{ }_{0} D_{x}^{\alpha} u(x)_{0} D_{x}^{\alpha} v(x)+{ }_{x} D_{T}^{\beta} u(x)_{x} D_{T}^{\beta} v(x) d x-\int_{0}^{T} f(x, u(x)) v(x) d x,
$$

for all $u, v \in \mathbb{H}_{0}^{\alpha, \beta}(0, T)$, which yields that

$$
\left\langle I^{\prime}(u), u\right\rangle=\int_{0}^{T}\left|{ }_{0} D_{x}^{\alpha} u(x)\right|^{2}+\left|{ }_{x} D_{T}^{\beta} u(x)\right|^{2} d x-\int_{0}^{T} f(x, u(x)) u(x) d x .
$$

Proof. Let

$$
I_{1}(u):=\frac{1}{2} \int_{0}^{T}\left[\left.\left.\right|_{0} D_{x}^{\alpha} u(x)\right|^{2}+\left.{ }_{x} D_{T}^{\beta} u(x)\right|^{2}\right] d x, \quad I_{2}(u):=\int_{0}^{T} F(x, u(x)) d x
$$

It is easy to check that $I_{1} \in C^{1}\left(\mathbb{H}_{0}^{\alpha, \beta}(0, T), \mathbb{R}\right)$, and we have

$$
\left\langle I_{1}^{\prime}(u), v\right\rangle=\int_{0}^{T}{ }_{0} D_{x}^{\alpha} u(x)_{0} D_{x}^{\alpha} v(x)+{ }_{x} D_{T}^{\beta} u(x){ }_{x} D_{T}^{\beta} v(x) d x .
$$

Therefore, it is sufficient to show that this is the case for $I_{2}$. By $\left(f_{1}\right)$, one has

$$
|F(x, \xi)| \leq b(x)|\xi|^{q}, \quad \forall(x, \xi) \in[0, T] \times \mathbb{R} .
$$

For any $u \in \mathbb{H}_{0}^{\alpha, \beta}(0, T)$, it follows from Remark 2.2 and (3.2) that

$$
\begin{aligned}
\int_{0}^{T}|F(x, u(x))| d x & \leq \int_{0}^{T} b(x)|u(x)|^{q} d x \leq\|b\|_{\infty}\|u\|_{L^{q}}^{q} \\
& \leq T^{\frac{2-q}{2}} \mathfrak{C}^{q}\|b\|_{\infty}\|u\|_{\alpha, \beta}^{q} .
\end{aligned}
$$

Therefore, $I$ defined by (1.4) is well defined on $\mathbb{H}_{0}^{\alpha, \beta}(0, T)$. 
Now, for any function $\theta:[0, T] \rightarrow(0,1)$, by $\left(f_{1}\right),(2.5)$ and the Hölder inequality, we have

$$
\begin{aligned}
& \int_{0}^{T} \max _{h \in[0,1]}|f(x, u(x)+\theta(x) h v(x)) v(x)| d x \leq \int_{0}^{T} \max _{h \in[0,1]}|f(x, u(x)+\theta(x) h v(x))||v(x)| d x \\
& \leq q\|b\|_{\infty} \int_{0}^{T}(|u(x)|+|v(x)|)^{q-1}|v(x)| d x \\
& \leq 2 q\|b\|_{\infty}\left[\left(\int_{0}^{T}|u(x)|^{2} d x\right)^{\frac{q-1}{2}}\left(\int_{0}^{T}|v(x)|^{\frac{2}{3-q}} d t\right)^{\frac{3-q}{2}}+\|v\|_{L^{q}}^{q}\right] \\
& \leq 2 q\|b\|_{\infty} T^{\frac{2-q}{2}} \mathfrak{C}^{q}\left(\|u\|_{\alpha, \beta}^{q-1}+\|v\|_{\alpha, \beta}^{q-1}\right)\|v\|_{\alpha, \beta}<+\infty .
\end{aligned}
$$

Let the functional $H: \mathbb{H}_{0}^{\alpha, \beta} \rightarrow \mathbb{R}$ defined by

$$
H(u)=\int_{0}^{T} F(x, u(x)) d x .
$$

Then, by the Mean Value Theorem, (3.4) and Lebesgue's Dominated Convergence Theorem, we have

$$
\lim _{h \rightarrow 0^{+}} \frac{H(u+h v)-H(u)}{h}=\lim _{h \rightarrow 0^{+}} \int_{0}^{T} f(x, u(x)+\theta(x) h v(x)) v(x) d x=\int_{0}^{T} f(x, u(x)) v(x) d x .
$$

Let's prove now that $H^{\prime}$ is continuous. Let $\left\{u_{n}\right\}_{n \in \mathbb{N}}, u \in \mathbb{H}_{0}^{\alpha, \beta}$ such that $u_{n} \rightarrow u$ strongly in $\mathbb{H}_{0}^{\alpha, \beta}$ as $n \rightarrow \infty$. Then $u_{n} \rightarrow u$ in $L^{2}[0, T]$, and so

$$
\lim _{n \rightarrow \infty} u_{n}(x)=u(x) \text {, a.e. } x \in \mathbb{R} .
$$

By $\left(f_{1}\right)$, for any bounded subinterval $\Omega \subset[0, T]$,

$$
\begin{aligned}
\int_{\Omega}\left|f\left(x, u_{n}(x)\right)\right|^{q^{\prime}} d x & \leq q^{q^{\prime}}\|b\|_{\infty}^{q^{\prime}} \int_{\Omega}\left|u_{n}(x)\right|^{(q-1) q^{\prime}} d x \\
& \leq q^{q^{\prime}} \mathfrak{C}^{q}\|b\|_{\infty}^{q^{\prime}}\left\|u_{n}\right\|_{\alpha, \beta}^{q}|\Omega|^{\frac{2-q}{2}} \leq C|\Omega|^{\frac{2-q}{2}} .
\end{aligned}
$$

It follows from (3.6) that the sequence $\left\{\left|f\left(t, u_{n}\right)-f(t, u)\right|^{q^{\prime}}\right\}$ is uniformly bounded and equiintegrable in $L^{1}(\Omega)$. The Vitali Convergence Theorem implies

$$
\lim _{n \rightarrow \infty} \int_{\Omega}\left|f\left(x, u_{n}(x)\right)-f(x, u(x))\right|^{q^{\prime}} d x=0
$$

So, by Hölder inequality and $(2.5)$, we obtain

$$
\begin{aligned}
& \left\|H^{\prime}\left(u_{n}\right)-H^{\prime}(u)\right\|_{\left(\mathbb{H}_{0}^{\alpha, \beta}\right)^{*}} \\
& =\sup _{v \in \mathbb{H}_{0}^{\alpha, \beta}(0, T),\|v\|_{\alpha, \beta}=1}\left|\int_{0}^{T}\left(f\left(x, u_{n}(x)\right)-f(x, u(x))\right) v(x) d x\right| \\
& \leq\left\|f\left(x, u_{n}\right)-f(x, u)\right\|_{L^{q^{\prime}}}\|v\|_{L^{q}} \\
& \leq \mathfrak{C} T^{\frac{2-q}{2}}\left\|f\left(x, u_{n}\right)-f(x, u)\right\|_{L^{q^{\prime}}} \rightarrow 0
\end{aligned}
$$


as $n \rightarrow \infty$. Therefore by $(3.5), I \in C^{1}\left(\mathbb{H}_{0}^{\alpha, \beta}(0, T), \mathbb{R}\right)$ and

$$
\left\langle I^{\prime}(u), v\right\rangle=\int_{0}^{T}{ }_{0} D_{x}^{\alpha} u(x){ }_{0} D_{x}^{\alpha} v(x)+{ }_{x} D_{T}^{\beta} u(x)_{x} D_{T}^{\beta} v(x) d x-\int_{0}^{T} f(x, u(x)) v(x) d x .
$$

Q.E.D.

Lemma 3.2. If $\left(f_{1}\right)$ and $\left(f_{2}\right)$ hold, then $I$ satisfies (PS)-condition.

Proof. Let $\left\{u_{n}\right\}_{n \in \mathbb{N}} \subset \mathbb{H}_{0}^{\alpha, \beta}(0, T)$ such that $\left\{I\left(u_{n}\right)\right\}$ is bounded and $I^{\prime}\left(u_{n}\right) \rightarrow 0$ as $n \rightarrow \infty$. Then there exists a constant $M>0$ such that

$$
\left|I\left(u_{n}\right)\right| \leq M \text { and }\left\|I^{\prime}\left(u_{n}\right)\right\|_{\left(\mathbb{H}_{0}^{\alpha, \beta}\right)^{*}} \leq M
$$

Therefore

$$
\begin{aligned}
\left(1-\frac{\mu}{2}\right)\left\|u_{n}\right\|_{\alpha, \beta}^{2} & =\left\langle I^{\prime}\left(u_{n}\right), u_{n}\right\rangle-\mu I\left(u_{n}\right) \\
& +\int_{0}^{T} f\left(t, u_{n}(t)\right) u_{n}(t)-\mu F\left(t, u_{n}(t)\right) d t \\
& \leq M\left\|u_{n}\right\|_{\alpha, \beta}+\mu M .
\end{aligned}
$$

Since $1<\mu<2,(3.8)$ shows that $\left\{u_{n}\right\}_{n \in \mathbb{N}}$ is bounded in $\mathbb{H}_{0}^{\alpha, \beta}(0, T)$. Since $\mathbb{H}_{0}^{\alpha, \beta}(0, T)$ is a reflexive space, up to a subsequence, still denote by $\left\{u_{n}\right\}_{n \in \mathbb{N}}$ such that $u_{n} \rightarrow u$ weakly in $\mathbb{H}_{0}^{\alpha, \beta}(0, T)$. Then

$$
\left\langle I^{\prime}\left(u_{n}\right)-I^{\prime}(u), u_{n}-u\right\rangle \rightarrow 0 .
$$

Moreover, by Theorem 2.3, up to a subsequence

$$
u_{n} \rightarrow u \text { strongly in } L^{2}(0, T) \text { and a.e. in }(0, T) .
$$

Thus, $f\left(x, u_{n}\right)\left(u_{n}-u\right) \rightarrow 0$ a.e. in $(0, T)$ as $n \rightarrow \infty$. Following the ideas of Lemma 3.1, it is easy to check that sequence $\left\{f\left(x, u_{n}\right)\left(u_{n}-u\right)\right\}$ is uniformly bounded and equi-integrable in $L^{1}(0, T)$. Hence, the Vitali Convergence Theorem implies

$$
\lim _{n \rightarrow \infty} \int_{0}^{T} f\left(x, u_{n}\right)\left(u_{n}-u\right) d x=0 .
$$

Consequently, combining (3.9), (3.10) with the following equality

$$
\left\langle I^{\prime}\left(u_{n}\right)-I^{\prime}(u), u_{n}-u\right\rangle=\left\|u_{n}-u\right\|_{\alpha, \beta}^{2}-\int_{0}^{T}\left(f\left(x, u_{n}\right)-f(x, u)\right)\left(u_{n}-u\right) d x,
$$

we deduce that $\left\|u_{k}-u\right\|_{\alpha, \beta} \rightarrow 0$ as $n \rightarrow+\infty$. That is, $I$ satisfies the (PS)-condition.

Q.E.D.

Remark 3.3. In view of Lemma $3.1, I \in C^{1}\left(\mathbb{H}_{0}^{\alpha, \beta}(0, T), \mathbb{R}\right)$ and by Lemma 3.2 , $I$ satisfies the (PS)-condition. Furthermore, by (3.3) we have

$$
\begin{aligned}
I(u) & =\frac{1}{2}\|u\|_{\alpha, \beta}^{2}-\int_{0}^{T} F(t, u(t)) d t \\
& \geq \frac{1}{2}\|u\|_{\alpha, \beta}^{2}-\mathfrak{C}^{q} T^{\frac{2-q}{2}}\|u\|_{\alpha, \beta}^{q} .
\end{aligned}
$$


Since $1<q<2$, (3.11) implies that $I(u) \rightarrow+\infty$ as $\|u\|_{\alpha, \beta} \rightarrow+\infty$. Consequently, $I$ is bounded from bellow. Therefore, by use of a standard minimizing argument (see Theorem 2.7 in [19]), $c=\inf _{\mathbb{H}_{0}^{\alpha, \beta}(0, T)} I(u)$ is a critical value of $I$, that is there exists a critical point $u^{*} \in \mathbb{H}_{0}^{\alpha, \beta}(0, T)$ such that

$$
I\left(u^{*}\right)=c .
$$

Furthermore, let $\varphi \in C_{0}^{\infty}[0, T]$ with $\|\varphi\|_{\alpha, \beta}=1$. Then, by $\left(f_{1}\right)$ we obtain that

$$
\begin{aligned}
I(\sigma \varphi) & =\frac{|\sigma|^{2}}{2}-\int_{0}^{T} F(t, \sigma \varphi(t)) d t \\
& \leq \frac{|\sigma|^{2}}{2}-|\sigma|^{q} \int_{\text {supp } \varphi} a(t)|\varphi(t)|^{q} d t,
\end{aligned}
$$

which yields that $I(\sigma \varphi)<0$ as $|\sigma|$ small enough because $1<q<2$. That is, the critical point $u^{*}$ is nontrivial.

The following result, whose proof can be seen Brezis [7], will play a key role in the proof of our main result

Theorem 3.4. Let $X$ be a separable and reflexive Banach space, then there exists $\left\{e_{n}\right\}_{n \in \mathbb{N}} \subset X$ and $\left\{e_{n}^{*}\right\}_{n \in \mathbb{N}} \subset X^{*}$ such that

$$
\left\langle e_{n}^{*}, e_{m}\right\rangle=\delta_{n, m}= \begin{cases}1 & \text { if } n=m \\ 0 & \text { if } n \neq m\end{cases}
$$

and

$$
X=\overline{\operatorname{span}\left\{e_{n} ; 1,2, \cdots\right\}} \text { and } X^{*}=\overline{\operatorname{span}\left\{e_{n}^{*} ; 1,2, \cdots\right\}} \text {. }
$$

Proof of Theorem 1.1. We note that, by $\left(f_{1}\right), I(0)=0$ and by $\left(f_{3}\right), I$ is an even functional. Denote by $\gamma(A)$ the genus of $A$. Set

$$
\begin{gathered}
\Sigma=\left\{A \subset E_{0}^{\alpha, p} \backslash\{0\}: \quad A \text { is closed in } E_{0}^{\alpha, p} \text { and symmetric with respect to } 0\right\}, \\
\Sigma_{k}=\{A \in \Sigma: \gamma(A) \geq k\}, \quad k=1,2, \cdots, \\
c_{k}=\inf _{A \in \Sigma_{k}} \sup _{u \in A} I(u), \quad k=1,2, \cdots,
\end{gathered}
$$

we have

$$
-\infty<c_{1} \leq c_{2} \leq \cdots \leq c_{k} \leq c_{k+1} \leq \cdots .
$$

Now, we will show that $c_{k}<0$ for every $k \in \mathbb{N}$. Since $\mathbb{H}_{0}^{\alpha, \beta}(0, T)$ is a reflexive and separable Hilbert space, consider $\left\{e_{n}\right\}_{n \in \mathbb{N}}$ a Schauder basis of $\mathbb{H}_{0}^{\alpha, \beta}(0, T)$ given by Theorem 3.4, and for each $k \in \mathbb{N}$, consider $X_{k}=\operatorname{span}\left\{e_{1}, e_{2}, \cdots, e_{k}\right\}$, the subspace of $\mathbb{H}_{0}^{\alpha, \beta}(0, T)$ generated by $k$ vectors $e_{1}, e_{2}, \cdots, e_{k}$. Since all norms of a finite dimensional normed space are equivalent, there exists a positive constant $C(k)$ which depends on $k$, such that

$$
-C(k)\|u\|_{\alpha, \beta}^{q} \geq-\int_{0}^{T}|u(t)|^{q} d t,
$$


for all $u \in X_{k}$. We now use $\left(f_{1}\right)$ to conclude that

$$
\begin{aligned}
I(u) & =\frac{1}{2}\|u\|_{\alpha, \beta}^{2}-\int_{0}^{T} F(t, u(t)) d t \\
& \leq \frac{1}{2}\|u\|_{\alpha, \beta}^{2}-\int_{0}^{T} a(t)|u(t)|^{q} d t \\
& \leq\|u\|_{\alpha, \beta}^{q}\left(\frac{1}{2}\|u\|_{\alpha, \beta}^{2-q}-\tilde{a} C(k)\right),
\end{aligned}
$$

where $\tilde{a}=\inf _{t \in[0, T]} a(t)$. Let $R$ be a positive constant such that

$$
\frac{1}{2} R^{2-q}<\tilde{a} C(k)
$$

So, for all $0<r<R$, and considering $K=\left\{u \in X_{k}:\|u\|_{\alpha, \beta}=r\right\}$, we get

$$
I(u) \leq r^{q}\left(\frac{1}{2} r^{2-q}-\tilde{a} C(k)\right)<R^{q}\left(\frac{1}{2} R^{2-q}-\tilde{a} C(k)\right)<0=I(0),
$$

which implies

$$
\sup _{K} I(u)<0=I(0)
$$

Since $X_{k}$ and $\mathbb{R}^{k}$ are isomorphic and $K$ and $S^{k-1}$ are homeomorphic, we conclude that $\gamma(K)=k$. Therefore, by the Clarke theorem, $I$ has at least $k$ pairs of different critical points. Since $k$ is arbitrary, we obtain infinitely many critical points of I.

Acknowledgements: I would like to show my great thanks to the reviewer for his/her carefully reading of the manuscript, giving valuable comments and suggestions to improve the exposition of the paper.

\section{References}

[1] Adams R. and Fournier J., Sobolev space, second ed., Academic Press, New York-London, 2003.

[2] Ambrosetti A. and Malchiodi A., Nonlinear analysis and semilinear elliptic problems, Cambridge Stud. Adv. Math. 14, 2007.

[3] Baleanu D., Güvenc Z. and Machado J. (eds), New trends in nanotechnology and fractional calculus applications, Singapore 2010.

[4] Belmekki M., Nieto J. and Rodríguez-López R., Existence of periodic solution for a nonlinear fractional differential equation, Bound. Value Probl. 2009, Art. ID 324561, 18 pp. (2009).

[5] Benchohra M., Cabada A. and Seba D., An existence result for nonlinear fractional differential equations on Banach spaces. Bound. Value Probl. 2009, Article ID 628916, 11 pp. (2009).

[6] Britton N., Reaction - Diffusion Equations and Their Applications to Biology, Academic Press Inc. London,1986. 
[7] Brezis H., Functional analysis, Sobolev spaces and partial differential equations, Springer, New York, 2011.

[8] Cantrell R. and Cosner C., Spatial ecology via reaction-diffusion equations, in: Wiley Series in Mathematical and Computational Biology, John Wiley \& Sons Ltd., Chichester, 2003.

[9] Clarke D., A variant of the Lusternik - Schnirelman theory, Indiana Univ. Math. J. 22, 65-74 (1972).

[10] Ervin V. and Roop J., Variational formulation for the stationary fractional advection dispersion equation, Numer. Meth. Part. Diff. Eqs, 22, 58-76(2006).

[11] Fazli H. and Bahrami F., On the steady solutions of fractional reaction-diffusion equations, Acceted manuscript in Filomat, 2016

[12] Jiao F. and Zhou Y., Existence of solution for a class of fractional boundary value problems via critical point theory. Comp. Math. Appl., 62, 1181-1199(2011).

[13] Jiao F. and Zhou Y., Existence results for fractional boundary value problem via critical point theory, Intern. Journal of Bif. and Chaos, 22(4), 1-17(2012).

[14] Kilbas A., Srivastava H. and Trujillo J., Theory and applications of fractional differential equations, North-Holland Mathematics Studies, vol 204, Amsterdam, 2006.

[15] Mawhin J. and Willen M., Critical point theory and Hamiltonian systems, Applied Mathematical Sciences 74, Springer, Berlin, 1989.

[16] Mendez A. and Torres C., Multiplicity of solutions for fractional Hamiltonian systems with Liouville-Weyl fractional derivarives, Fract. Calc. Appl. Anal., 18, No 4, 875-890, 2015.

[17] Nyamoradi N., Infinitely Many Solutions for a Class of Fractional Boundary Value Problems with Dirichlet Boundary Conditions, Medit. J. Math., 11(1), 75-87(2014).

[18] Podlubny I., Fractional differential equations, Academic Press, New York, 1999.

[19] Rabinowitz P., Minimax method in critical point theory with applications to differential equations, CBMS Amer. Math. Soc., No 65, 1986.

[20] Rivero M., Trujillo J., Vázquez L. and Velasco M., Fractional dynamics of populations, Appl. Math. Comput, 218, 1089 - 1095(2011).

[21] Sabatier J., Agrawal O. and Tenreiro Machado J., Advances in fractional calculus. Theoretical developments and applications in physics and engineering, Springer-Verlag, Berlin, 2007.

[22] Samko S., Kilbas A. and Marichev O. Fractional integrals and derivatives: Theory and applications, Gordon and Breach, New York, 1993.

[23] Schechter M., Linking methods in critical point theory, Birkhäuser, Boston, 1999.

[24] Sokolov I. and Klafter J., From diffusion to anomalous diffusion: A century after Einsteins Brownian motion, Chaos, 15, 2, 26-103(2005). 
[25] Torres C., Existence of solution for fractional Hamiltonian systems, Electronic Jour. Diff. Eq. 2013, 259, 1-12(2013).

[26] Torres C., Mountain pass solution for a fractional boundary value problem, Journal of Fractional Calculus and Applications, 5, 1, 1-10(2014).

[27] Torres C., Existence of a solution for fractional forced pendulum, Journal of Applied Mathematics and Computational Mechanics, 13, 1, 125-142(2014).

[28] Torres C. Boundary value problem with fractional p-Laplacian operator, Adv. Nonlinear Anal. (2015) (Preprint), doi: 10.1515/anona-2015-0076.

[29] Torres C., Ground state solution for a class of differential equations with left and right fractional derivatives, Math. Methods Appl. Sci, 38, 5063-5073(2015).

[30] Torres C., Existence and symmetric result for Liouville-Weyl fractional nonlinear Schrödinger equation, Commun Nonlinear Sci Numer Simulat., 27, 314-327(2015).

[31] Torres C., Existence of solution for fractional Langevin equation: variational approach, Electron. J. Qual. Theory Differ. Equ. 2014, No 54, 1-14.

[32] Xu J., O'Regan D. and Zhang K., Multiple solutions for a class of fractional Hamiltonian systems, Fract. Calc. Appl. Anal., 18, No 1, 48-63(2015)

[33] Zhang Z. and Li J., Variational approach to solutions for a class of fractional boundary value problems, Electron. J. Qual. Theory Differ. Equ. 2015, No 11, 1-10.

[34] Zhou Y., Basic theory of fractional differential equations, World Scientific Publishing Co. Pte. Ltd. 2014. 